\title{
Case series of precursor B-cell lymphoblastic lymphoma
}

\author{
Jin Yong Kim ${ }^{1}$, Sang Yong $\mathrm{Om}^{1}$, Su-Jin Shin ${ }^{2}$, Jeong Eun $\mathrm{Kim}^{3}$, Dok Hyun Yoon ${ }^{3}$, Cheolwon Suh ${ }^{3}$ \\ Departments of ${ }^{1}$ Internal Medicine, ${ }^{2}$ Pathology, ${ }^{3}$ Oncology, Asan Medical Center, University of Ulsan College of Medicine, Seoul, \\ Korea
}

p-ISSN 2287-979X / e-ISSN 2288-0011 http://dx.doi.org/10.5045/br.2014.49.4.270 Blood Res 2014;49:270-4.

Received on November 18, 2012

Revised on October 20, 2014

Accepted on October 28, 2014

\section{Correspondence to}

Cheolwon Suh, M.D., Ph.D.

Department of Oncology, Asan Medical

Center, University of Ulsan College of

Medicine, 88, Olympic-ro 43-gil,

Songpa-gu, Seoul 138-736, Korea

Tel: $+82-2-3010-3209$

Fax: $+82-2-3010-6961$

E-mail: csuh@amc.seoul.kr

\section{Abstract}

Precursor B-cell lymphoblastic lymphoma (B-LBL) is an uncommon subtype of Non-Hodgkin lymphoma (NHL), accounting for only $0.3 \%$ of $\mathrm{NHL}$ in adults and less than $10 \%$ of all LBL cases. Unlike T-cell LBL, it usually presents with extranodal involvement while sparing the bone marrow (BM). Among the 27 patients with LBL treated in the Asan Medical Center between January 2007 and March 2012, 3 had B-LBL. All had a good performance status and low International Prognostic Index. However, unlike most previously reported cases, the patients had lymphoma in their bone marrow and extranodal sites such as bone and lung. After intensive combination chemotherapy, one patient achieved a complete response and the other 2 patients, a partial response. Our experience suggests that multiple extranodal sites may be involved in B-LBL and BM involvement may not be as infrequent as previously thought. Furthermore, intensive chemotherapy seems to be effective.

Key Words B-cell lymphoblastic lymphoma, Chemotherapy, Bone marrow involvement

(C) 2014 Korean Society of Hematology

\section{INTRODUCTION}

Lymphoblastic lymphoma (LBL) accounts for approximately $2 \%$ of all non-Hodgkin lymphoma (NHL) [1], and approximately $90 \%$ are of immature T-cell lineage [2]. Precursor B-cell LBL (B-LBL) is uncommon and accounts for less than $10 \%$ of total LBL cases and $0.3 \%$ of adult NHL $[1,3]$.

The term LBL is used when the disease is confined to mass lesions including the lymph nodes and extranodal organs with no or minimal evidence of bone marrow (BM) involvement [4]. The disease presents mainly with extranodal involvement, sparing the BM. The most commonly involved sites include the skin (33\%), lymph nodes (22\%), bone (19\%), and mediastinum (5\%), while hepatomegaly, splenomegaly, a leukemic picture, or involvement of the central nervous system have been reported infrequently [5-7]. The natural history of adult B-LBL has not been well defined because of its rarity.

From January 2007 to March 2012, 1,230 adult patients presented with NHL at the Asan Medical Center, Seoul, Korea. Among the 27 (2.2\%) patients diagnosed with LBL, only $3(0.2 \%)$ had B-LBL. In this report, we aimed to summa- rize the clinical characteristics and clinical course of these 3 B-LBL cases.

\section{CASE REPORT}

\section{Case I}

A 46-year-old woman presented with progressive pain in her left hip joint without any systemic symptoms. Physical examination and laboratory findings were unremarkable. Bone single photon emission computed tomography (SPECT) showed increased uptake in the left acetabulum. Magnetic resonance imaging (MRI) showed an ill-defined heterogeneous enhancement from the left acetabulum to the iliac bone (Fig. 1A). No other involvement sites were found with further investigations including neck, chest, abdominopelvic computed tomography (CT), and whole body 18-Fluoro-deoxyglucose positron emission tomography (FDG PET; Fig. 1B). A biopsy of the left acetabulum was performed. Positive immunohistochemical (IHC) staining for CD20 and PAX5 and negative staining for CD3, CD34, CD56, CD117, and terminal deoxynucleotidyl transferase (TdT) were diagnostic for B-LBL (Fig. 2). The BM biopsy had 1\% neoplastic lymphocytes. Conventional cytogenetics performed on a BM 

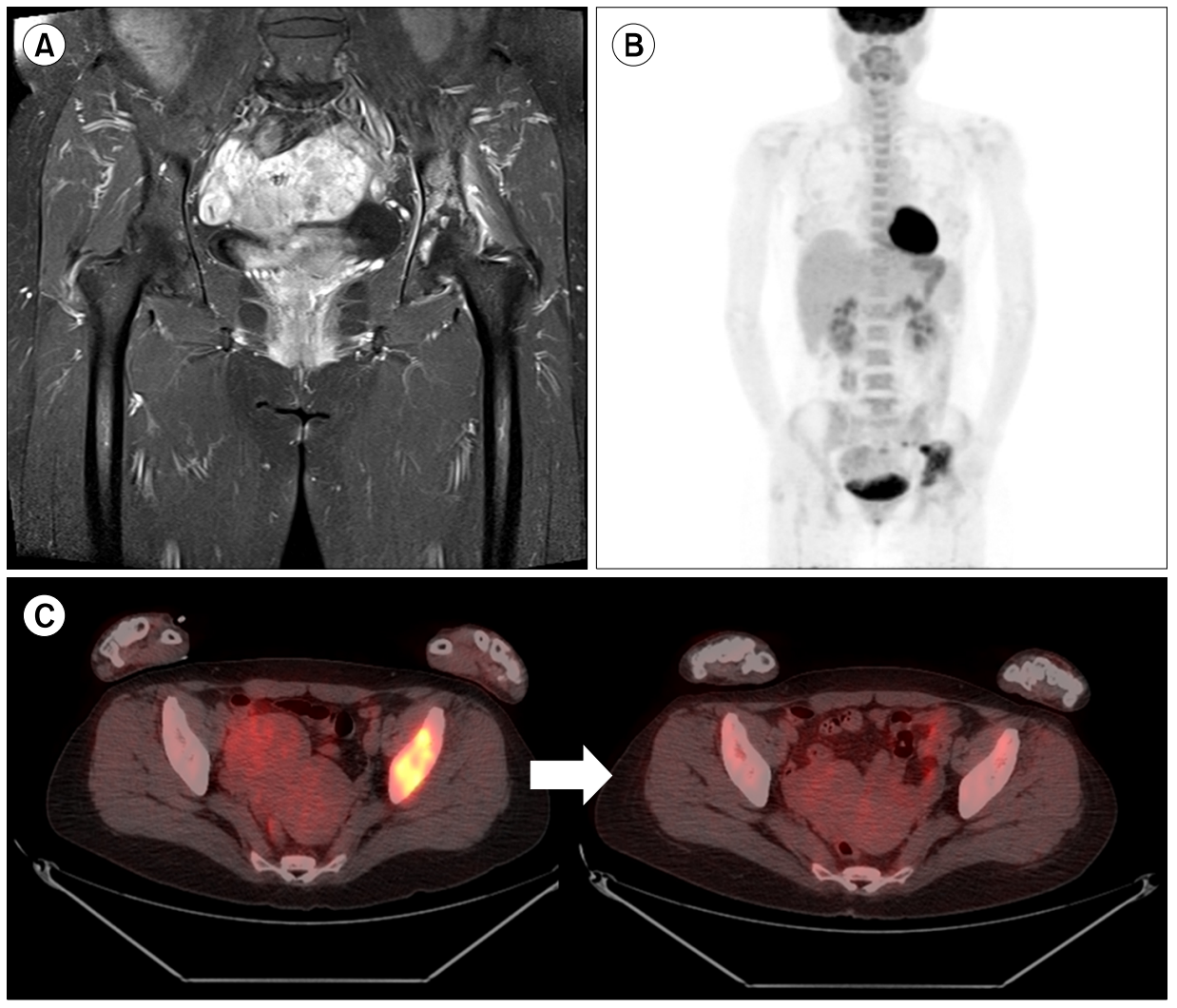

Fig. 1. (A) Post-contrast T2weighted coronal pelvic magnetic resonance image showing a high T2 signal and enhancement in the left acetabulum and iliac bone. (B) A positron emission tomography (PET) image showing inhomogeneous hypermetabolic activity (maximum SUV=5.6) in the left ilium and acetabulum. (C) PET image showing the disappearance of a hypermetabolic lesion in the left ilium and acetabulum after vincristine, methylprednisolone, daunorubicin, and L-asparaginase (VPDL) induction chemotherapy.
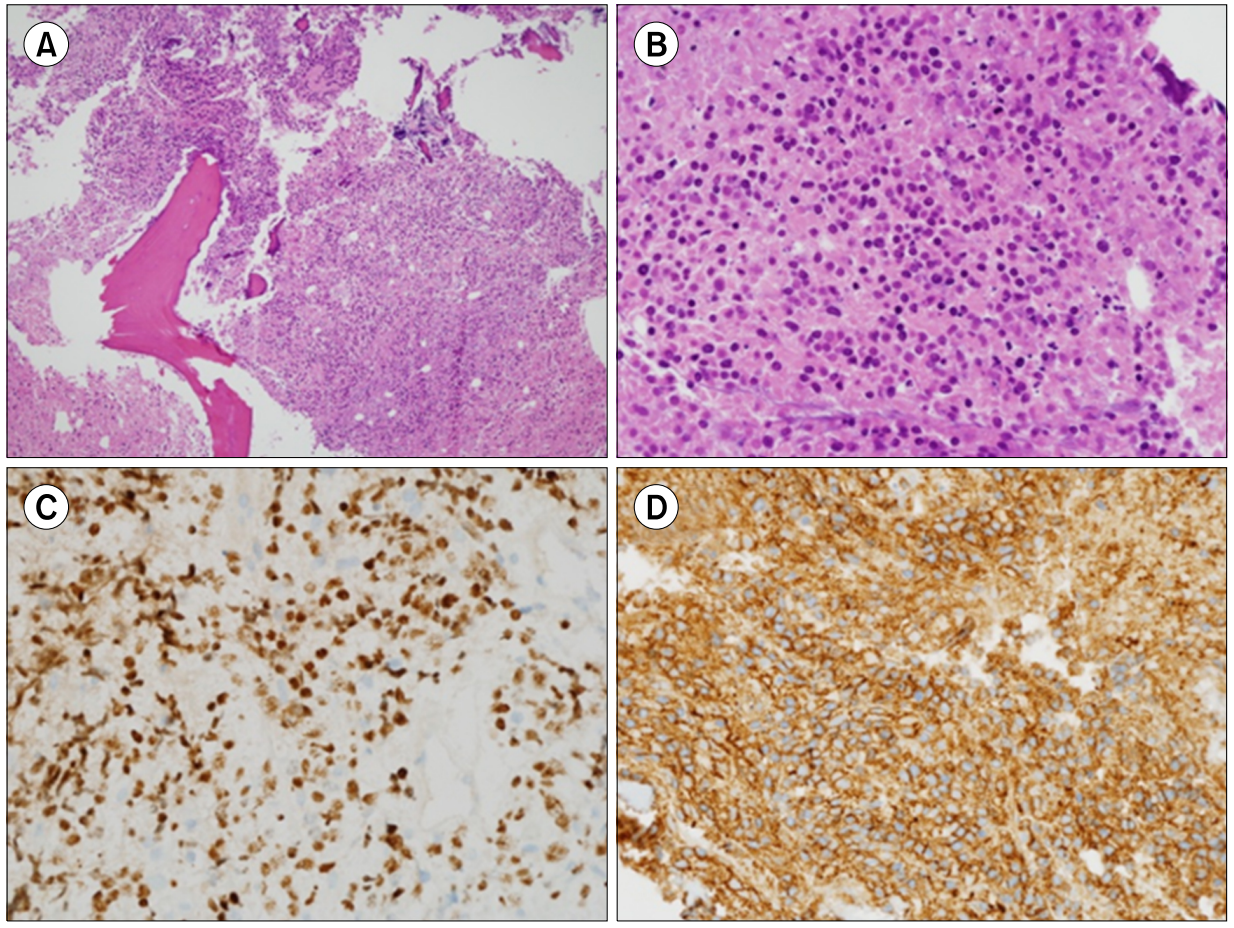

Fig. 2. (A) Microscope analysis of the biopsy specimen revealing diffuse infiltration of small-tomedium sized lymphocytes with massive necrosis $(\times 100$; hematoxylin and eosin [H\&E]). (B) High magnification shows monotonous tumor cells with a moderate amount of cytoplasm, round-tooval shaped indented nuclei, condensed chromatin and inconspicuous nucleoli (lymphoblast), and nuclear debris with necrosis ( $\times 400$; H\&E). (C) The neoplastic cells show nuclear staining for PAX5, and (D) diffuse membranous staining for CD20.

aspirate revealed a normal karyotype. Serum lactate dehydrogenase $(\mathrm{LDH})$ was within normal limits (184 IU/L) at diagnosis. According to the Ann Arbor classification, the patient had stage IV disease.

The patient achieved complete remission (CR) after 2 cycles of vincristine, methylprednisolone, daunorubicin, and 

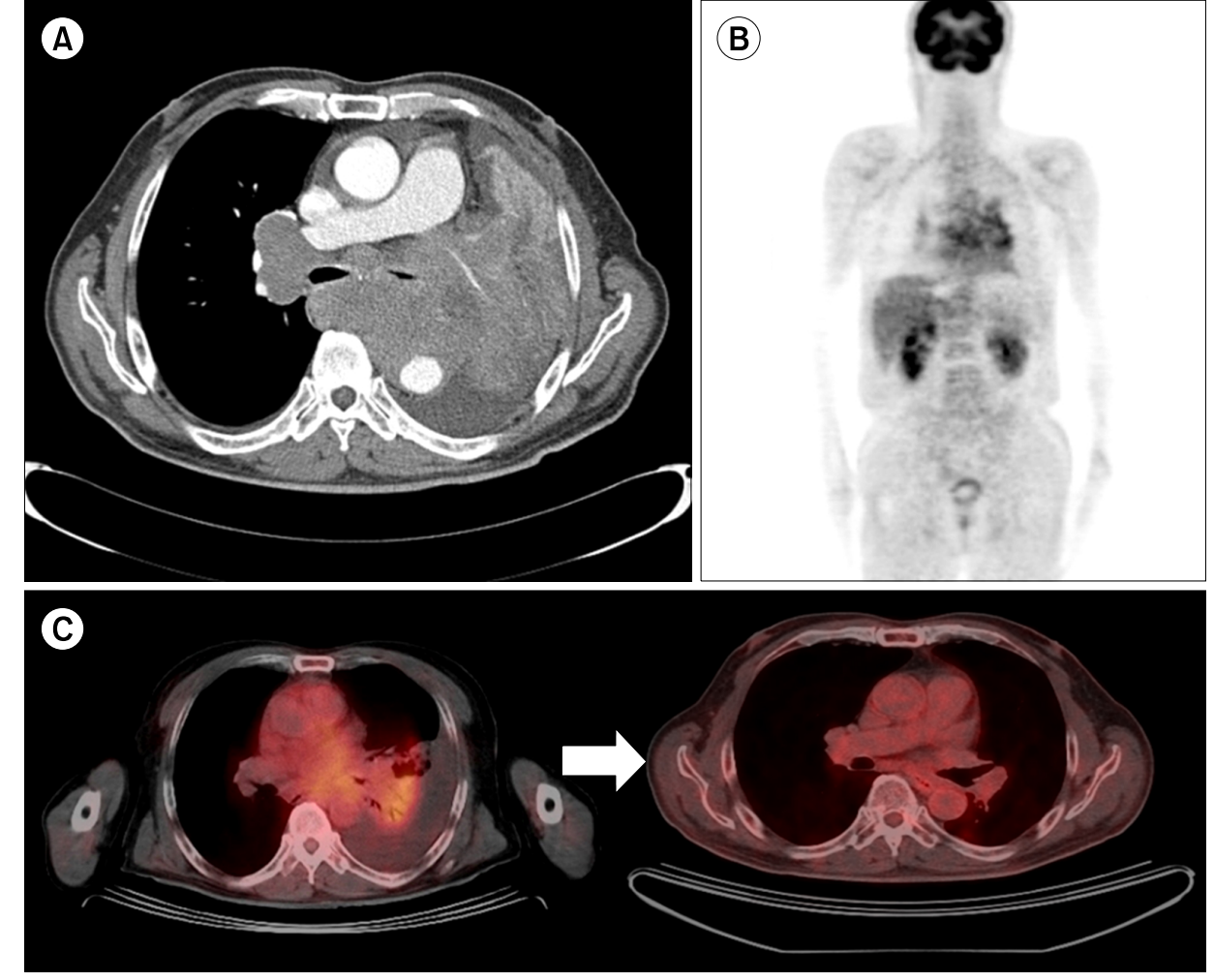

Fig. 3. (A) Chest computed tomography showing conglomerated enlarged lymph nodes in the bilateral mediastinum causing compression of the central airway, and encasement of the left pulmonary artery and descending aorta. (B) A positron emission tomography (PET) image showing a hypermetabolic central lung mass in the left lung with direct invasion of the mediastinum and post-obstructive atelectasis. (C) PET image showing decreased extent and activity of hypermetabolic lesions after rituximab, cyclophosphamide, doxorubicin, vincristine, and prednisolone (R-CHOP) chemotherapy.
L-asparaginase (VPDL) chemotherapy, [8] and was still receiving treatment on the data cut-off date.

\section{Case 2}

A 65-year-old man presented with a 2-month history of fever, cough, sputum, and dyspnea on exertion. He had undergone a chest CT at a primary clinic, which revealed a lung mass, conglomerated enlarged lymph nodes in the bilateral mediastinum compressing the central airway, with encasement of the left pulmonary artery and descending thoracic aorta, and pericardial and pleural effusion (Fig. 3A and B). A lymph node biopsy was performed that was initially suggestive of small cell carcinoma; therefore, the patient received concurrent chemoradiation with etoposide and carboplatin for 3 days. Then, he was referred to our hospital for a second opinion. A review of the biopsy slides suggested that the specimen had been insufficient to make a definitive diagnosis and a rebiopsy was required. An endoscopic ultrasound-guided biopsy was made of the same mesenteric lymph node. He was diagnosed with diffuse large B-cell lymphoma (DLBCL); the BM biopsy also revealed involvement of DLBCL with a normal karyotype. Serum LDH was $260 \mathrm{IU} / \mathrm{L}$ at diagnosis. The patient had stage IV disease according to the Ann Arbor classification. He received 1 cycle of rituximab, cyclophosphamide, doxorubicin, vincristine, and prednisolone (R-CHOP) chemotherapy. Thereafter, the diagnosis was revised to B-LBL based on positive IHC staining for leukocyte common antigen (LCA), CD20, CD10, MUM1, and TdT, and negative staining for CD5, CD23, CD56, BCL-6, cytokeratin, TTF-1, and cyclin D1 (Fig. 4). Despite the new diagnosis, the patient, who was a Jehovah's Witness, insisted on continuing with $\mathrm{R}-\mathrm{CHOP}$ to avoid the increased risk of blood transfusion associated with VPDL chemotherapy. Although he achieved a partial response (PR) after 6 R-CHOP cycles (Fig. 3C), the disease progressed 2 months after treatment. The patient refused salvage chemotherapy and was lost to follow-up. However, he was found to be alive at the data cut-off date with an overall survival of 27 months according to data from the National Health Insurance Corporation of Korea.

\section{Case 3}

A 44-year-old woman presented with multiple palpable masses in her neck, but without any systemic symptoms. Laboratory tests revealed a WBC count of $11 \times 10^{9} / \mathrm{L}, \mathrm{Hb}$ level of $11.4 \mathrm{~g} / \mathrm{dL}$, platelet count of $194 \times 10^{9} / \mathrm{L}$, and LDH level of $624 \mathrm{IU} / \mathrm{L}$, with $72 \%$ blast cells in her peripheral blood smear.

A CT scan showed multiple enlarged lymph nodes in the neck, in the left supraclavicular and infraclavicular areas, right paratracheal areas, and in the subcarinal and inguinal areas. The left supraclavicular lymph node was biopsied, and the patient was diagnosed with B-LBL, with positive IHC staining for CD10, PAX5, TdT, and BCL-2, and negative staining for CD3, CD5, and CD20. The BM biopsy had 61.6\% lymphoblasts. According to the Ann Arbor classification, the patient had stage IV disease. She achieved PR after induction VPDL chemotherapy and was still receiving treat- 

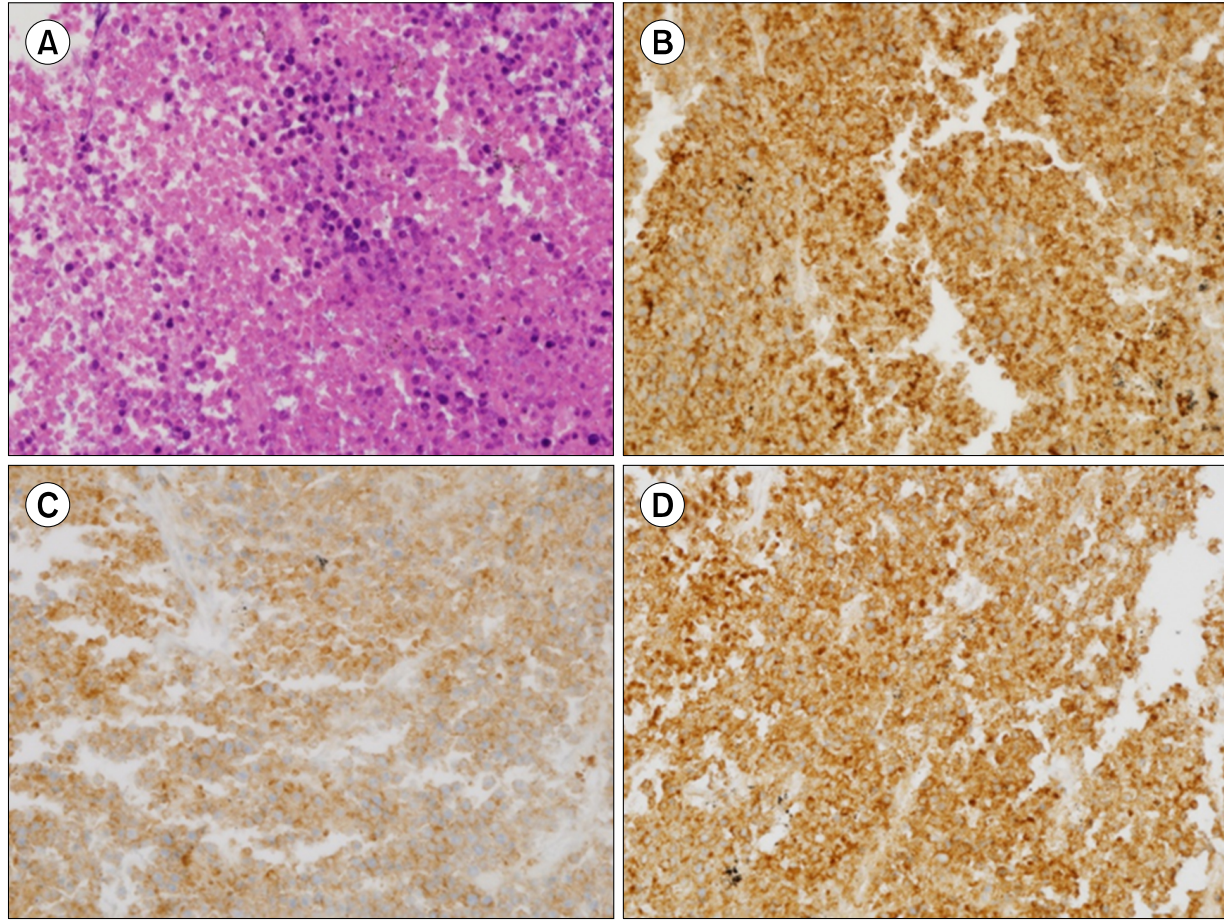

Fig. 4. (A) High magnification shows diffuse infiltration of monotonous tumor cells with a moderate amount of cytoplasm, roundto-oval shaped indented nuclei, condensed chromatin and inconspicuous nucleoli (lymphoblast), and nuclear debris with necrosis $(\times 400$; hematoxylin and eosin). (B) The neoplastic cells show diffuse membranous staining for CD20, (C) cytoplasmic staining for CD10, and (D) diffuse membranous staining for leukocyte common antigen (LCA). ment on the data cutoff date.

\section{DISCUSSION}

B-LBL, which constitutes less than $10 \%$ of all LBL, very rarely presents with $\mathrm{BM}$ involvement [1]. In this report of 3 B-LBL cases, all had stage IV disease, good performance status, and a low International Prognostic Index (IPI) score (Table 1). These clinical features were similar to those observed in another report, also from Korea [4]. In contrast, however, 2 patients had lymphoma involvement at multiple extranodal sites including the acetabulum and lung, and BM involvement was noted in all patients.

T-LBL patients, compared to those with B-LBL, are known to be younger and have a higher rate of mediastinal tumors or $\mathrm{BM}$ involvement $[3,9]$. In the vast majority of B-LBL patients, mediastinal masses and $\mathrm{BM}$ involvement are rare, but lymph nodes and extranodal sites, such as skin, bone, and soft tissue are more frequently involved than in T-cell LBL (T-LBL) [3, 5, 9]. In most cases, B-LBL and T-LBL cannot be distinguished on histologic features alone without immunophenotyping [10].

In B-LBL, tumor cells are virtually always positive for B cell markers CD19, CD22, and CD79a. In most cases, they are also positive for CD10, CD24, PAX5, and TdT, while the expression of CD20 and CD34 is variable [9]. In case 1 presented here, small malignant cells were positive for CD20 and PAX5; in case 2, they were positive for CD10, CD20, LCA, and TdT; and in case 3 , they were positive for CD10, PAX5, and TdT. These IHC findings were con-
Table 1. Characteristics of patients.

\begin{tabular}{llll}
\hline & \multicolumn{1}{c}{ Case 1 } & \multicolumn{1}{c}{ Case 2 } & Case 3 \\
\hline Age/Gender & $46 / \mathrm{F}$ & $65 / \mathrm{M}$ & $44 / \mathrm{F}$ \\
Ann Arbor stage & IV & IV & IV \\
Serum LDH (IU/L) & 184 & 260 & 624 \\
B symptoms & No & No & No \\
Extranodal involvement & Acetabulum & Lung & No \\
Bone marrow involvement & Yes & Yes & Yes \\
IPI & 2 & 2 & 2 \\
Treatment & VPDL & R-CHOP & VPDL \\
Response after treatment & CR & PR & PR \\
Survival & Alive, 2 mo & Alive, 27 mo & Alive \\
\hline
\end{tabular}

Abbreviations: $\mathrm{CR}$, complete response; $\mathrm{F}$, female; IPI, international prognostic index; IU, international units; $M$, male; PR, partial response; R-CHOP, rituximab, cyclophosphamide, doxorubicin, vincristine, and prednisolone; VPDL, vincristine, methylprednisolone, daunorubicin, and L-asparaginase.

sistent with B-LBL. In case 3, the laboratory findings, which included mild anemia and a normal platelet count (with the normal range of megakaryocytes in the BM biopsy) supported the diagnosis of B-LBL with leukemic manifestation, rather than B-ALL.

Of the 2 patients who received VPDL chemotherapy [8, 11], 1 achieved CR and the other PR. Although case 2 received $1 \mathrm{R}$-CHOP cycle because of the initial misdiagnosis, he also achieved PR after 6 R-CHOP cycles. As LBL is highly aggressive, intensive multi-drug leukemia chemotherapy protocols are usually recommended [9]. Intensive protocols designed for aggressive NHL improve the CR rate, but survival 
is poorer than that obtained using regimens designed for other aggressive lymphomas [12]. Improvements in longterm LBL outcome have been achieved with ALL-type regimens [13]. As chemotherapy intensity correlates with outcome in LBL, more intensive NHL regimens fare better than conventional NHL regimens [14].

In summary, the cases presented indicate that B-LBL can involve multiple extranodal sites and BM involvement may not be as infrequent as previously reported. Furthermore, intensive chemotherapy seems to be effective in this disease.

\section{Authors' Disclosures of Potential Conflicts of Interest}

No potential conflicts of interest relevant to this article were reported.

\section{REFERENCES}

1. The Non-Hodgkin's Lymphoma Classification Project. A clinical evaluation of the International Lymphoma Study Group classification of non-Hodgkin's lymphoma. Blood 1997;89:3909-18.

2. Pui CH, Behm FG, Crist WM. Clinical and biologic relevance of immunologic marker studies in childhood acute lymphoblastic leukemia. Blood 1993;82:343-62.

3. Soslow RA, Baergen RN, Warnke RA. B-lineage lymphoblastic lymphoma is a clinicopathologic entity distinct from other histologically similar aggressive lymphomas with blastic morphology. Cancer 1999;85:2648-54.

4. Chang MH, Kim SJ, Kim K, et al. Clinical features and treatment outcomes of adult B- and T-lymphoblastic lymphoma: results of multicentre analysis in Korea. Leuk Lymphoma 2009;50:1119-25.

5. Lin P, Jones D, Dorfman DM, Medeiros LJ. Precursor B-cell lymphoblastic lymphoma: a predominantly extranodal tumor with low propensity for leukemic involvement. Am J Surg Pathol 2000;24:1480-90.

6. Kaygusuz I, Toptas T, Guven A, Firatli-Tuglular T, Tecimer T, Bayik M. Precursor B cell lymphoblastic lymphoma presenting as a solitary bone tumor: a case report and review of the literature. Int J Hematol 2010;92:757-61.

7. Maitra A, McKenna RW, Weinberg AG, Schneider NR, Kroft SH. Precursor B-cell lymphoblastic lymphoma. A study of nine cases lacking blood and bone marrow involvement and review of the literature. Am J Clin Pathol 2001;115:868-75.

8. Linker CA, Levitt LJ, O'Donnell M, Forman SJ, Ries CA. Treatment of adult acute lymphoblastic leukemia with intensive cyclical chemotherapy: a follow-up report. Blood 1991;78:2814-22.

9. Cortelazzo S, Ponzoni M, Ferreri AJ, Hoelzer D. Lymphoblastic lymphoma. Crit Rev Oncol Hematol 2011;79:330-43.

10. Soslow RA, Bhargava V, Warnke RA. IC2, TdT, bcl-2, and CD34 expression in paraffin-embedded high-grade lymphoma/acute lymphoblastic leukemia distinguishes between distinct clinicopathologic entities. Hum Pathol 1997;28:1158-65.

11. Yoon DH, Sohn BS, Lee WJ, et al. VPDL chemotherapy for T-cell lymphoblastic lymphoma (T-LBL) in adults: comparison with upfront autologous stem cell transplantation in a single center. Korean J Hematol 2008;43:138-44.

12. Le Gouill S, Lepretre S, Briere J, et al. Adult lymphoblastic lymphoma: a retrospective analysis of 92 patients under 61 years included in the LNH87/93 trials. Leukemia 2003;17:2220-4.

13. Reiter A, Schrappe M, Ludwig WD, et al. Intensive ALL-type therapy without local radiotherapy provides a $90 \%$ event-free survival for children with T-cell lymphoblastic lymphoma: a BFM group report. Blood 2000;95:416-21.

14. Willemze R, Zijlmans JM, den Ottolander GJ, et al. High-dose Ara-C for remission induction and consolidation of previously untreated adults with ALL or lymphoblastic lymphoma. Ann Hematol 1995;70:71-4. 